by other agents, are referred to as a sensitization even though the biochemical basis of the reaction is explained by Truitt and Walshe in volume 1 . This is unfortunate because true allergic hypersensitivity to alcoholic beverages is a known clinical condition, and no reference to this syndrome appears in either volume. Numerous scattered references appear to the actions of drugs. As an example, Von Wartberg states that ambiguous results have been reported from clinical studies with metronidazole and tells the reader to see volume 1 . However, there are no further references to this drug in the index of either volume. None of the chapters in either volume deals exclusively with the relationship between alcohol and drug action.

Volumes 1 and 2 of the Biology of Alcoholism contain much worthwhile accumulated information in a series of review articles in which the facts and references may be repeated on several occasions. As a book, however, it lacks the integration and cohesion to make it into a comprehensive reference manual. The individual articles provide good reviews of aspects of the biology of alcoholism but there are gaps in the subject matter. The indexes are insufficiently detailed to enable the reader easily to select the relevant facts about the subjects in which he is interested. These volumes provide an expensive method of obtaining a series of good review articles by experts on their subjects between four covers. They will provide worthwhile additions to reference libraries. C. W. M. WILSON

\section{Mutation Research}

Chemical Mutagenesis in Mammals and Man. Edited by F. Vogel and G. Rohrborn. Pp. xiv +519 . (Springer : Berlin and New York, 1970.) 124 DM; $\$ 34.10$.

This book contains valuable chapters on mutation research contributed at a symposium held in Maine in 1969, and several additional chapters thereafter. In the preface, the editors provide an overall masterly introduction to the material covered in the book. The first three chapters deal with molecular mechanisms involved in the production of spontaneous or induced mutations and also on the potential impact of chemical mutagens in the human environment. The following fourteen chapters describe research methodologies, such as dominant lethal test, in vivo cytogenetic studies and hostmediated assay for practical screening of potential chemical mutagens. Systems for detecting the induction of recessive mutations and chromosome aberrations in mammalian somatic cell cultures, including man, are also dealt with. Even though the main aim of this book is to emphasize methodologies for those who are concerned with the problem of chemical mutagenesis in relation to man, various results obtained to date with available methods on some chemical groups, such as alkylating agents, antimetabolites, acridines and purines, are described in the next ten chapters. All in all, this book is an important addition toward the understanding of many problems relating to chemical mutagenesis.

\section{SAMUEL S. EpSTEIN}

\section{Vertebrate Physiology}

How Animals Work. By Knut SchmidtNielsen. Pp. vi+114. (Cambridge University: London, July 1972.) £2.20; $\$ 5.95$.

BASED on the Hans Gadow Lectures for 1970-71 at the University of Cambridge, Professor Schmidt-Nielsen's latest book is aimed chiefly at undergraduate students of vertebrate physiology: it merits a far wider audience. Indeed, it is a pleasure to find quite complex concepts explained with such beguiling simplicity and lucidity. In spite of its broad title, this little volume is directly concerned with those aspects of vertebrate physiology to which the author and his colleagues have made such valuable contributions during the last two decades or so, especially in relation to desert adaptation. It is thus an expression of Knut SchmidtNielsen's personal views; but these are conveyed with such conviction that even his sternest critics must be swayed by the power and reason of his arguments.

This incisive account first illustrates how the principle of countercurrents has been invoked to reduce water-loss through evaporation in the respiration of kangaroo-rats. A temperature gradient is established and heat exchange results in the temperature of the exhaled air approaching that of the ambient air (according to the principles of the Stirling and Carnot heat engines). The legs of wading birds and the flippers of whales are next cited as further examples of countercurrent heat exchange. The problem of losing heat by panting is now discussed, and attention turned to the respiration of birds. New evidence from the author's laboratory suggests that air flows unidirectionally through the bird lung, against a countercurrent of blood. (A similar system occurs in the fish gill, but is not possible in the lungs of mammals.) This mechanism enables birds to migrate for great distances without excessive water-loss, and to fly at altitudes so great that mammals would barely be able to survive in them. Since respiration is synchronized with wing beat, tidal volume in trumpeter swans must be adjusted to a low frequency. A large dead space is therefore necessary to achieve a suitable concentration of carbon dioxide in the posterior air-sacs which comprise the mixing chambers, and this is provided by the elongated trachea.

The expenditure of energy in locomotion is next discussed and the need for animals to use evaporation of water for keeping cool. The principle of countercurrents in the carotid rete enables their heads to remain cool, even though the bodies of gazelles and antelopes may reach temperatures over $46^{\circ} \mathrm{C}$. Independent control of temperature by the same means in limited parts of the body is responsible for the high muscle temperatures of tuna fishes and sharks. Such countercurrent systems utilize vascular channels of the millimetre size range, which means that they cannot inadvertently function as gas exchangers.

The book next compares such exchangers with multipliers like the swim bladders of fishes (which can secrete against pressures of several hundred atmospheres) and the mammalian kidney. It concludes with a discussion of problems of scaling. Schmidt-Nielsen clearly vindicates the opinions of those zoologists who believe that university teaching ought to be original and to provide exciting and stimulating ideas. More is needed than merely to provide a ésumé of facts culled from the textbooks and reviews currently fashionable. Carl Pantin would surely have approved of Schmidt-Nielsen's lectures. J. L. Cloudsley-Thompson

\section{Discriminating Enzymes}

Stereochemistry and its Application in Biochemistry: The Relation Between Substrate Symmetry and Biological Stereospecificity. By William L. Alworth. Pp. xi+311. (Wiley: New York and London, August 1972.) $£ 7.75$.

This book sets out to show, to any student or practitioner of biochemistry who can lay hands on a set of molecular models, why a reaction proceeding on an enzyme can often discriminate absolutely between two groups (in the same molecule) which would be attacked with precisely the same probability by ordinary, symmetrical chemical reagents. The aim has been achieved: anyone prepared to study the text with attention and a modicum of intellectual effort will be able to grasp the author's explanations and to apply them. Indeed, there may be a different risk: an intending reader might think that a subject requiring so much explanation must be intrinsically difficult. To him, I would say: "Don't be afraid ; all this discrimination and specificity of enzymes arises simply from their power (just like yours with molecular models) to distinguish 